Mirian Carmita Arcentales-Fajardo; Darwin Gabriel García-Herrera; Nancy Marcela Cárdenas-Cordero; Juan Carlos Erazo-Álvarez

DOI 10.35381/cm.v6i3.394

\title{
Ambiente Educativo, Trabajo Colaborativo Docente y por áreas en Educación General Básica Media
}

\section{Educational environment, teaching collaborative work and by areas in middle basic general education}

\author{
Felipe Abelardo Alba-Contreras \\ felipe.alba.07@est.ucacue.edu.ec \\ Universidad Católica de Cuenca, Azogues \\ Ecuador \\ https://orcid.org/0000-0003-0058-5152 \\ Darwin Gabriel García-Herrera \\ dggarciah@ucacue.edu.ec \\ Universidad Católica de Cuenca, Azogues \\ Ecuador \\ https://orcid.org/0000-0001-6813-8100 \\ Luis Bolívar Cabrera-Berrezueta \\ bolivarcabrera@ucacue.edu.ec \\ Universidad Católica de Cuenca, Cuenca \\ Ecuador \\ https://orcid.org/0000-0002-6853-635X \\ Juan Carlos Erazo-Álvarez \\ jcerazo@ucacue.edu.ec \\ Universidad Católica de Cuenca, Cuenca \\ Ecuador \\ https://orcid.org/0000-0001-6480-2270
}

Recibido: 20 de agosto de 2020

Aprobado: 15 de noviembre de 2020 


\title{
RESUMEN
}

La investigación tiene como objetivo analizar la ejecución de la propuesta de trabajo por áreas con docentes especialistas en las áreas de conocimiento mediante un trabajo colaborativo en el subnivel de Básica Media de la Unidad Educativa Agronómico Salesiano, en la cual se utilizó una metodología mixta y de cohorte transversal, que a través del uso de técnicas de recolección de información, como la entrevista y la encuesta que fueron aplicadas a la totalidad del universo que conforman dicho subnivel de enseñanza, se pudo medir el nivel de satisfacción de todos los actores educativos frente a la propuesta implementada, datos que fueron analizados con la ayuda del programa IBM SPSS, con la utilización de tablas de frecuencia y el Chi cuadrado de Pearson, obteniendo resultados satisfactorios en todas la variables analizadas, pero con la posibilidad de mejorar algunos aspectos, los cuales fueron abordados en la propuesta.

Descriptores: Aprendizaje activo; autoaprendizaje; método de aprendizaje. (Palabras tomadas del Tesauro UNESCO)

\begin{abstract}
The research aims to analyze the execution of the work proposal by areas with specialist teachers in the areas of knowledge through collaborative work at the Sub-level of Basic Medium of the Salesian Agronomic Educational Unit, in which a mixed methodology and of cross-sectional cohort, that through the use of information collection techniques, such as the interview and the survey that were applied to the entire universe that make up said sub-level of education, it was possible to measure the level of satisfaction of all educational actors with respect to the implemented proposal, data that were analyzed with the help of the IBM SPSS program, with the use of frequency tables and Pearson's Chi square, obtaining satisfactory results in all the variables analyzed, but with the possibility of improving some aspects, which were addressed in the proposal.
\end{abstract}

Descriptors: Activity learning; self instruction self instruction; learning methods. (Words taken from UNESCO Thesaurus). 
Universidad Nacional Experimental Francisco de Miranda (UNEFM). Santa Ana de Coro. Venezuela

Mirian Carmita Arcentales-Fajardo; Darwin Gabriel García-Herrera; Nancy Marcela Cárdenas-Cordero; Juan Carlos Erazo-Álvarez

\section{INTRODUCCIÓN}

La Educación General Básica es muy importante en el proceso formativo integral de los niños y niñas del mundo, en estos subniveles la mayor parte de los centros educativos, por no decir todos, trabajan con un docente por aula o salón de clase; el profesional de la educación aborda todas las áreas de aprendizaje y debe garantizar cumplir con los objetivos del currículo educativo vigente en cada uno de los países, fomentando en cada uno de los destinatarios el desarrollo del pensamiento crítico y reflexivo, en un ambiente de convivencia armónico.

Para (Escobar Medina, 2015) la base fundamental de la educación es la interactividad entre el docente y el alumno, en el proceso de enseñanza aprendizaje, esta cumple una función de enlace, que facilita la adquisición de los conocimientos y que estos sean significativos, permitiéndole al estudiante que pueda manifestarse con lo que piensa y siente; generando un grupo social en el aula de clases en donde se escucha y comprende a los demás, involucrando a todos en esta interactividad y motivando a que sean partícipes de la misma.

Otro aspecto importante dentro del proceso de enseñanza - aprendizaje, es la flexibilidad y el tacto pedagógico del Docente, que contribuye a forjar un ambiente afectivo y sentimental empático con los estudiantes, que también incide positivamente en la interactividad educativa y trasciende en la vida de ellos, lo que significa que la labor del Docente educador no solo se limita a conseguir los objetivos de aprendizaje, sino que también aporta en el desarrollo afectivo y social del alumno, habilidades que serán de utilidad para relacionarse, asumir reglas, compromisos, capacidad de negociar y adaptarse a su realidad y contexto.

El problema de este proceso de interactividad educativa, es que los alumnos poseen características específicas, cada uno tiene un ritmo de aprendizaje distinto, diversas necesidades que no siempre pueden ser atendidas y solucionadas, por un solo docente de aula, esto conlleva a que la comunicación no fluya con esa intencionalidad antes 
Universidad Nacional Experimental Francisco de Miranda (UNEFM). Santa Ana de Coro. Venezuela

Mirian Carmita Arcentales-Fajardo; Darwin Gabriel García-Herrera; Nancy Marcela Cárdenas-Cordero; Juan Carlos Erazo-Álvarez

mencionada, convirtiendo a docente y estudiante en simples receptores y emisores de mensajes, provocando una comunicación deficiente sin una trascendencia interactiva motivadora que coadyuve el conseguir ese objetivo educativo integral, peor aún, si en el proceso educativo encontramos Docentes autoritarios, intransigentes, que abusen de su posición, y debiliten la educación. (OREALC/UNESCO Santiago, 2013) dice que el profesionalismo y la capacidad Docente es lo primordial para alcanzar la calidad educativa, si los estudiantes no encuentran en sus escuelas oportunidades que sean significativas para el proceso de enseñanza aprendizaje, no incitará dicha calidad.

Por otro lado, (Barrera Erreyes, Barragán García, \& Ortega Zurita, 2017) manifiestan que, la educación y la formación es parte del ser humano, por lo tanto, todos los días se aprende en interacción con: en las instituciones con los compañeros, en la sociedad y con el docente que transmite conocimientos científicos y una formación humanista a niños y jóvenes. Sin embargo, el problema que persiste dentro del sistema educativo ecuatoriano es que no existe una coherencia de estructura entre todos los niveles a pesar de contar con La Actualización Curricular 2016.

Los estudiantes evaluados por el Instituto Nacional de Evaluación Educativa [INEVAL,2017] en conocimientos y actitudes básicas obtienen un promedio estándar de $7.44 / 10$, esa responsabilidad no se le atribuye a los docentes y su labor, sino a que aún no existe una verdadera articulación de las mallas curriculares de cada subnivel, por consiguiente, este estudio tiene como resultados que los actores principales de la educación, son quienes deben realizar esa interconexión necesaria entre el pensum de estudios de cada uno de los subniveles.

Así entonces, el MINEDUC (Ministerio de Educación, 2020) tiene explícito en el Currículo de Básica Media que los estudiantes, con la guía del docente de aula, alcanzarán las habilidades y competencias en áreas de aprendizaje básico, de cultura, deporte, apreciando características interculturales y respetando su cultura. Sin embargo, con 
Universidad Nacional Experimental Francisco de Miranda (UNEFM). Santa Ana de Coro. Venezuela

Mirian Carmita Arcentales-Fajardo; Darwin Gabriel García-Herrera; Nancy Marcela Cárdenas-Cordero; Juan Carlos Erazo-Álvarez

todas esas condiciones, se ha podido identificar que en la Unidad Educativa Agronómico Salesiano:

Los estudiantes de educación general básica media (5to, 6to y 7 mo año ), pierden el interés y la atención por su formación académica al pasar todo el tiempo con el mismo docente, que organiza sus clases con la misma metodología, planificación y técnicas de evaluación en todas las áreas básicas, provocando que, el educando disminuye su motivación y la participación dentro del aula; a su vez, el docente no alcanza a dar atención a todos los estudiantes, a sus necesidades y ritmos de aprendizaje, teniendo como consecuencia algún rezago en el aprendizaje del estudiante. El trabajo del docente se torna individualizado y no colaborativo, la interacción alumno - docente se ve afectada con el pasar del tiempo por la monotonía, decayendo en la consecución de los objetivos de aprendizaje.

Por las razones antes mencionadas se plantea la implementación de un modelo de gestión diferente a la propuesta curricular vigente del subnivel básica media, en la cual los docentes se especialicen en cada área de conocimiento, de tal forma que, bajo una organización pedagógica y trabajo colaborativo, cada profesor sea quien genere su ambiente educativo, propendiendo a que el proceso de enseñanza - aprendizaje sea más atractivo, activo ,lúdico, significativo y estimule a los estudiantes a tener una participación más interactiva dentro de la institución, y de manera específica en su salón de clases.

\section{Referencial teórico}

En el presente artículo se propone una nueva posibilidad de trabajo en el subnivel de educación General Básica Media por lo tanto, se parte de una revisión bibliográfica que no pretende ser profunda, pero sí que ponga de manifiesto los elementos más relevantes sobre un ambiente educativo saludable y pertinente, que permita un proceso de enseñanza aprendizaje significativo; por consiguiente en la investigación de (Castro Pérez \& Morales Ramírez, 2015) se expone que, el ambiente educativo debe ir más allá 
Universidad Nacional Experimental Francisco de Miranda (UNEFM). Santa Ana de Coro. Venezuela

Mirian Carmita Arcentales-Fajardo; Darwin Gabriel García-Herrera; Nancy Marcela Cárdenas-Cordero; Juan Carlos Erazo-Álvarez

de contar con un espacio físico y contemplar las relaciones interpersonales dentro de la actividad de enseñanza aprendizaje.

Así también menciona sobre la importancia de buscar nuevas estrategias que posibiliten la creación de espacios educativos con características motivadoras, estimulantes, eficientes e integradoras, en la cual se presente particularmente ese escenario dinámico donde exista y se genere condiciones para el aprendizaje, desarrollando capacidades, competencias, habilidades y valores en los niños y niñas, a través de procesos que involucran acciones, experiencias y vivencias socio afectivas con cada uno de los actores educativos.

En el ambiente educativo se toma en cuenta toda la organización y disposición de los espacios, la relación que se establece entre diferentes elementos, con ciertos lineamientos de comportamiento que en él se desarrollan, lo más importante, la interrelación e interactividad que se produce entre las personas, y el papel que cumple cada uno de ellos en los diferentes propósitos educativos, demostrando que es necesario tener un ambiente que incite el aprendizaje y el desarrollo integral de los niños y niñas de cada institución.

Para que el proceso de enseñanza - aprendizaje sea eficiente, es importante tener en cuenta los objetivos educativos con base a las necesidades y los intereses de los niños y niñas, de esta manera es el docente quien tiene en sus manos la posibilidad de convertir su actividad pedagógica en lo más acertado y adecuado para satisfacer los requerimientos educativos y ritmos de aprendizaje de los estudiantes, tomando las mejores decisiones para el logro y consecución de una formación crítica, reflexiva e integral de los mismos.

Por otra parte, no se puede dejar de lado la organización de los ambientes de aprendizaje que resulten llamativos e interesantes para los estudiantes, pues justamente es ahí donde se potencian con calidad sus aptitudes y actitudes, logrando solventar todas las necesidades que ellos muestran, cabe mencionar que en la instauración de este cambio, 
Universidad Nacional Experimental Francisco de Miranda (UNEFM). Santa Ana de Coro. Venezuela

Mirian Carmita Arcentales-Fajardo; Darwin Gabriel García-Herrera; Nancy Marcela Cárdenas-Cordero; Juan Carlos Erazo-Álvarez

se requiere considerar varios factores que componen este ambiente, como lo es: la infraestructura, instalaciones, espacios, materiales de trabajo didáctico, pero las de mayor importancia incidencia son: la capacitación docente, el currículo, la buenas prácticas educativas y la interactividad entre docente y estudiante.

Según (Cruz Aguilar, 2020) esta interactividad pedagógica, esa relación con el otro, entre el docente y el niño ha tenido mejores resultados cuando contiene elementos de cuidado, diálogo que permite aprender conjuntamente, preocupación por el bienestar de cada uno de ellos y un seguimiento y acompañamiento en el proceso de aprendizaje, un clima de afectividad en el entorno educativo que promueve que los niños sean felices, ambientes sin violencia, en donde los niños y niñas realicen sus actividades académicas sin presiones, ni tensiones, con la posibilidad de avanzar a su propio ritmo, en donde prime la motivación, y la interacción que permita el trabajo colectivo y colaborativo.

\section{Trabajo Colaborativo.}

De acuerdo con (Krichesky \& Murillo, 2018) el trabajo colaborativo docente es una alternativa metodológica que apuesta por el cambio en la educación, resulta muy importante que los docentes se reúnan, hablen y planifiquen nuevas estrategias que causen impacto en el proceso de enseñanza aprendizaje y a través de nuevos conocimientos se geste propuestas de innovación y mejora escolar, por lo tanto, la colaboración promovería procesos y prácticas pedagógicas significativas e innovadoras en la actividad educativa; el identificar la forma de trabajo colectivo que promueva estos cambios positivos es la labor de muchas investigaciones.

Así, se manifiesta que la educación mira con mucho interés a la aplicación de esta metodología colaborativa entre docentes, porque supone una forma dinámica de cimentar una organización de trabajo colectivo, ordenado y coherente que tiene como uno de los fines el aprendizaje y por ende la mejora escolar, no obstante, otro elemento fundamental según (Zubizarreta, García - Ruiz, \& López , 2018) son los padres de familia; su 
Universidad Nacional Experimental Francisco de Miranda (UNEFM). Santa Ana de Coro. Venezuela

Mirian Carmita Arcentales-Fajardo; Darwin Gabriel García-Herrera; Nancy Marcela Cárdenas-Cordero; Juan Carlos Erazo-Álvarez

participación en la escuela mejora el rendimiento de los niños, aportan también con planes y proyectos que tiene como objetivo el desarrollo integral del niño como actor principal de la educación.

Sin embargo, a pesar de las grandes posibilidades que puede generar la aplicación de esta metodología se tiene que recalcar que la implementación y el desarrollo de la misma en las escuelas no es tarea fácil, influyen varios aspectos, que va desde las condiciones físicas en las instituciones educativas, así como también la poca o nula formación y capacitación docente que aporte a la adquisición de destrezas que permitan apropiarse del trabajo colaborativo, por ello, será la escasa capacidad de llegar a acuerdos y de negociar posiciones diferentes, que limitarían el proceso.

Por lo tanto, es necesario que se conozca de manera precisa las condiciones para que la colaboración resulte una herramienta estratégica para los docentes, misma que deberá ser instaurada en el aprendizaje y la innovación; son muchos los estudios que corroboran que la colaboración genera nuevas posibilidades de desarrollo profesional, (Liesa, Castelló, \& Becerril, 2018) manifiestan que las nuevas prácticas generan nuevos aprendizajes, autonomía en los estudiantes como la capacidad para tomar decisiones, en base a conocimientos, actitudes y valores, por eso es necesario que los docentes cambien su estructura en cuanto a la práctica de enseñanza - aprendizaje y así no solo generen aprendizajes entre alumnos sino también entre los docentes

Desde esta perspectiva, los docentes pueden aprender a partir de sus diversas experiencias, constituyéndose así la metodología colaborativa en una herramienta para mejorar los procesos de enseñanza, ya que, en esos intercambios e interacción se proporcionan elementos pedagógicos que se pueden aplicar dentro del aula de clases, que resultarán motivadores e incitarán a tomar decisiones acertadas, desde una visión compartida en beneficio de los estudiantes, cabe resaltar que la intervención de los directivos es muy importante por cuanto ellos son los que presten las condiciones necesarias para la promoción del trabajo colaborativo. 
Universidad Nacional Experimental Francisco de Miranda (UNEFM). Santa Ana de Coro. Venezuela

Mirian Carmita Arcentales-Fajardo; Darwin Gabriel García-Herrera; Nancy Marcela Cárdenas-Cordero; Juan Carlos Erazo-Álvarez

De esta forma, como resultado obtendremos experiencias satisfactorias en la comunicación entre docentes para la resolución de conflictos, lo que favorece en mantener un clima saludable en la escuela, además les permite consensuar material pedagógico, buenas prácticas en el aula y al mismo tiempo compartir y utilizar recursos didácticos, una forma de gestión que reduce la carga administrativa y permite desarrollar en conjunto el trabajo interdisciplinario entre diferentes áreas de conocimiento, enlazando contenidos y programando actividades de aprendizaje.

\section{Aprendizaje Colaborativo}

Desde otro punto de vista, según (González Vargas, 2014) el trabajo colaborativo posee muchas características, las cuales le dan varios significados que van asociados a una diversidad de conductas, valores, habitos, normas que los docentes en su interacción pueden compartir para fortalecer su quehacer educativo, por lo tanto, su significado no es limitado a un simple trabajo colectivo, este conlleva procesos de negociación, reflexión y gestión convirtiendola en una herramienta que engloba muchas ventajas para el desarrollo de la enseñanza.

Es significativo exponer también que dentro de las instituciones educativas existen representaciones sociales que son de trascendental importancia, que ilustran a los docentes de situaciones y experiencias que se viven en el día a día, lo que acontece en el ambiente de trabajo y la comunicación que fluye en la misma, lo que significa que cada docente se forma así mismo a partir de esa interactividad y relación con los otros, convirtiéndose en un proceso dinámico y continuo que construye conocimiento desde la realidad contextual, histórica y cultural.

Según (Oliveira Sá, 2017) el trabajo en pares de los docentes es un método eficaz para edificar prácticas de enseñanza reflexivas, que hacen que el aprendizaje de los estudiantes sea exitoso, así como el desarrollo profesional del docente dentro del aula de clase, este método permite que el docente sea quien analice con su colega o par su 
Universidad Nacional Experimental Francisco de Miranda (UNEFM). Santa Ana de Coro. Venezuela

Mirian Carmita Arcentales-Fajardo; Darwin Gabriel García-Herrera; Nancy Marcela Cárdenas-Cordero; Juan Carlos Erazo-Álvarez

propia práctica profesional, logrando mejores resultados que coger un curso de capacitación, mejorando aspectos curriculares, manejo de los estudiantes en el aula, trabajo colaborativo docente, promoviendo el trabajo colaborativo en los estudiantes, diversificación de recursos en el aula y actitud proactiva en la enseñanza.

Otro punto de análisis es la organización de la institución, considerando que cada una tiene su particularidad y la hace diferente de otras, con características propias de contexto, forma de vida, convirtiéndose de este modo en espacios auténticos en el cual se desarrolla y establece la actividad docente, y toda interacción que busca alcanzar los objetivos escolares, en este sentido no se puede negar de ninguna manera que los docentes son los actores principales de la formación y la innovación, a quienes se les atribuye la gran tarea de promover el trabajo colaborativo dentro de la escuela.

\section{Organización escolar.}

La organización escolar es fundamental para la buena marcha de las instituciones educativas, para (Fuentes Sordo, 2015) esta organización debe estar alineada con lo que la sociedad requiera, por consiguiente, sus fines y objetivos deben responder a las mismas, relacionando los elementos y los factores que están comprometidos; como unidad esencial el docente, el cual está supeditado a las disposiciones y normativas institucionales que permitan el afianzamiento de un proceso sistematico y coherente en lo pedagógico y didáctico, a fin de conseguir resultados efectivos.

$\mathrm{Si}$ los componentes que conforman la organización escolar no funcionan como un sistema, es posible que los resultados sean ineficientes, es así que, el currículo debe ser implementado de tal manera que responda al contexto y a los diferentes escenarios, para dar respuesta a las necesidades que presenta la comunidad educativa, por eso es indispensable tener claro lo que supone la organización en la actividad escolar, diseño curricular, orgánico funcional, nuevas metodologías, uso de recursos, y planes estratégicos, etc. 
Universidad Nacional Experimental Francisco de Miranda (UNEFM). Santa Ana de Coro. Venezuela

Mirian Carmita Arcentales-Fajardo; Darwin Gabriel García-Herrera; Nancy Marcela Cárdenas-Cordero; Juan Carlos Erazo-Álvarez

El proceso sistemático de la organización escolar y el orden de la gestión de la actividad pedagógica educativa institucional, serán pilares fundamentales en el cumplimiento de las metas y objetivos planteados en favor de los estudiantes y su formación académica integral, los logros de una buena dirección administrativa lo determina sin duda la organización escolar, que deberá considerar las características que tienen los estudiantes para poder potenciar el desarrollo de sus habilidades, actitudes, aptitudes y valores.

(Amorim, 2017) manifiesta que en la organización escolar debe existir un líder educativo, quien es el que va a tomar las decisiones en la institución y asegurar un ambiente pedagógico innovador, relaciones humanas saludables, una educación de calidad con nuevos saberes, nuevas metodologías, conocimientos que se actualiza frecuentemente y promueva la investigación. Una organización que rompa el individualismo y genere el trabajo en equipo, colaborativo para un ambiente escolar científico, cultural, social, emocional, pedagógico y diversificado.

De la misma forma (Ramírez-Cardona , Calderón-Hernández, \& Castaño-Duque, 2015) a través de su investigación indica que la gestión educativa en la organización escolar debe constituirse en un proceso dinámico, integrador y que se adapte a los cambios, para en el desarrollo poder mejorarlas y no limitarlas a guías que denoten cierto egoísmo, sino que respondan más bien a objetivos bien planteados con el fin de conseguir la formación de excelentes seres humanos, por ello, esta planeación debe ser construida por todo los actores educativos bajo la consideración de algunas dimensiones.

La calidad educativa, la organización escolar, y el rol que cumplen los docentes, son dimensiones encaminadas a la consolidación de los objetivos educativos, estas se perfilan a conseguir el desarrollo de las potencialidades del individuo, siempre con miras a que su inclusión en la sociedad sea con sentido equitativo y competencias productivas, ese resultado que se espera conseguir seria la derivación de tener objetivos 
Universidad Nacional Experimental Francisco de Miranda (UNEFM). Santa Ana de Coro. Venezuela

Mirian Carmita Arcentales-Fajardo; Darwin Gabriel García-Herrera; Nancy Marcela Cárdenas-Cordero; Juan Carlos Erazo-Álvarez

institucionales bien planteados, una educación inclusiva, equitativa, que aproveche los recursos y se fundamente en una eficiente organización y propuesta académica.

Finalmente se aborda el rol que cumplen los individuos en la institución educativa, quizá esta dimensión sea la más importante de todas porque se habla del componente humano quien acciona los demás dimensiones, puesto que son los constructores, los que analizan y reformulan las organizaciones educativas, que en cierto momento pueden ser vistos de una forma solo participativa de los procesos que esta organización escolar requiere, pero en realidad son los que están involucrados en la marcha diaria del quehacer educativo.

En esta investigación se toca aspectos muy significativos para la educación es así que (Bailey Moreno, Flores Fahara, \& González Rivera, 2016) evidencia que las creencias de los maestros, podrían llegar a ser una forma de que la perspectiva de la enseñanza cambie, se conoce que la influencia de otras personas en cuanto a experiencias compartidas cobran un sustancial sentido en su formación y en la manera de analizar y evaluar los conocimientos, en este sentido por la importancia que tienen las creencias, los docentes actúan entorno a ello en el proceso de enseñanza.

No obstante, se señala que las creencias de los maestros que están ejerciendo inciden en la aceptación de nuevos esquemas pedagógicos o lineamientos educativos, estas modificaciones educativas requieren de cambios que los docentes no van adoptar fácilmente en su práctica de enseñanza aprendizaje, se recalca que las creencias de los maestros, funcionan como filtro y buscan dar relevancia a las situaciones vividas y compartidas de tal forma que se adapten y den sentido a lo que realiza dentro de su actividad educativa.

De este modo, conociendo que las creencias de los maestros se mantienen y predominan por sobre toda clase de cambio pedagógico y programas de formación, se debe pensar en que para que estos permitan un cambio de estructura de creencias y se genere nuevos aprendizajes, deben tener un alto grado de motivación que les introduzca a realizar una 
Universidad Nacional Experimental Francisco de Miranda (UNEFM). Santa Ana de Coro. Venezuela

Mirian Carmita Arcentales-Fajardo; Darwin Gabriel García-Herrera; Nancy Marcela Cárdenas-Cordero; Juan Carlos Erazo-Álvarez

reflexión individual y colaborativa entre docentes, este proceso hará que ellos sean los que propongan nuevos procesos de aprendizaje.

Se puede pensar que posiblemente la calidad educativa este afectada por cambios y reformas resueltas a nivel central, por las autoridades, dejando de lado al personal docente causando en ellos desmotivación y por ende una ejecución de procesos educativos deficientes, desistiendo de las posibilidades de cambio de modelo educativo como el que se propone en esta investigación, y salir de ese academicismo, y convertir la educación en un proceso motivador tanto para el docente y el estudiante, que se encuentran buscando nuevas formas de realizar una práctica de enseñanza eficaz y eficiente.

\section{Currículo}

En la actualidad y en la sociedad en la que nos desenvolvemos es necesario que tomemos en cuenta las exigencias de los niños, niñas y jóvenes en cuanto a educación nos referimos con planteamientos innovadores y flexibles que den respuestas apropiadas, a través de un sistema educativo de calidad que integre a todos, adaptándose a los cambios acelerados que se da en aspectos de conocimiento, comunicación y tecnología, lo que hoy en día se conoce como cultura globalizada, que nos obliga de cierto modo a buscar nuevas formas de prácticas educativas.

El Ecuador tiene un sistema educativo escolarizado, con una población estudiantil que va desde los 4 hasta los 17 años, los mismos se encuentran distribuidos en los niveles de, preparatoria, general básica y bachillerato así lo manifiesta el Ministerio de Educación (MINEDUC, 2020) por lo que es responsabilidad de directivos y docentes dar respuesta a las nececidades que presentan esa gran diversidad de estudiantes que buscan alcanzar los objetivos planteados en el sistema educativo, promoviendo el desarrollo de sus potencialidades y capacidades, siempre y cuando se logre el apoyo en la 
Universidad Nacional Experimental Francisco de Miranda (UNEFM). Santa Ana de Coro. Venezuela

Mirian Carmita Arcentales-Fajardo; Darwin Gabriel García-Herrera; Nancy Marcela Cárdenas-Cordero; Juan Carlos Erazo-Álvarez

organización escolar y fundamentalmente en los docentes quienes estarán directamente involucrados en el proceso académico.

En este sentido el currículo es la herramienta que guía nuestro accionar institucional, asi como a cada una de las áreas de conocimiento, en las cuales se precisa las destrezas y contenidos que se abordara en el proceso de enseñanza aprendizaje, engloban las experiencias que serán tratadas en los diferentes niveles, en las aulas y con los estudiantes especificamente, entonces con certeza se puede decir que es la institucion educativa en donde se desarrolla de manera práctica la educación que el Estado pone a disposicion para todos.

Sin embargo, se debe tomar en cuenta que las instituciones son las que directamente deben dar respuesta a las exigencias educativas, por lo tanto esto requiere de que se tenga cierta autonomía, para lo que se refiere a organización institucional y la propuesta curricular, que permita adecuar una atención pertinente a los estudiantes, atender toda esa diversidad, mediante un trabajo organizado, inclusivo y colaborativo de los maestros, y, estaríamos abriendo paso a una verdadera calidad educativa.

Una población estudiantil tan diversa, debe ser atendida mediante la diversificación de recursos y del talento humano, es decir los docentes, para que surja verdaderos cambios en el sistema educativo, con el diseño de un modelo acorde y adecuado al educando, que sea reflejo de la propuesta educativa institucional, misma que busca la formación axiológica del estudiantado y la integridad social en la escuela que articule totalmente el currículo para la consolidación de los objetivos de aprendizaje.

Partiendo de lo manifestado anteriormente, (Herrera Pavo \& Cochancela Patiño, 2020) nos muestra cual ha sido el proceso evolutivo de la educación en Ecuador, desde la reforma de 1996 hasta la actualización y fortalecimiento curricular del 2016, modelo curricular vigente que ha demostrado que en este transcurso las mencionadas reformas han tratado de adaptarse a las realidades sociales, económica, políticas, tecnológicas; como se ve, va mucho más allá de poseer los contenidos de aprendizaje, pues ha 
Universidad Nacional Experimental Francisco de Miranda (UNEFM). Santa Ana de Coro. Venezuela

Mirian Carmita Arcentales-Fajardo; Darwin Gabriel García-Herrera; Nancy Marcela Cárdenas-Cordero; Juan Carlos Erazo-Álvarez

buscado constituirse como una guía, con las orientaciones y los elementos necesarios para cumplir los objetivos planteados en las instituciones educativas y en las aulas de clase.

Pese a ello, el componente social ha hecho que se sobrecargue la propuesta curricular, con contenidos diversos en las áreas de conocimiento y con los ejes transversales que responden a las mencionadas nuevas necesidades sociales, lo que ha configurado el currículo con destrezas con criterio de desempeño básicas para cada subnivel, y las destrezas con criterio de desempeño imprescindibles, mismas que podrán ser alcanzadas a lo largo de su formación académica.

Este enfoque socioconstructivista, permite cambiar la perspectiva de la responsabilidad de diseño de la propuesta curricular, y propone una responsabilidad compartida, en donde el Estado es quien estructura el currículo general, que llega a las instituciones educativas para su revisión y complemento en la propuesta curricular institucional, a fin de que desde cada área de conocimiento los docentes puedan adaptar a la realidad de su aula y a los grupos de estudiantes correspondientes, mediante la elaboración de sus planificaciones, cumpliendo de esta forma los tres niveles de concreción curricular (macro, meso y micro).

\section{Currículo 2016 Básica Media.}

Para la presente investigación es pertinente tomar el artículo 2, literal w de La Ley Orgánica de Educación Intercultural (Ministerio de Educación, 2016). Además (Ministerio de Educación, 2020), en su apartado de la propuesta curricular para la básica media, nos otorga algunos lineamientos de flexibilidad del mismo para poder contextualizar el trabajo académico y responder a la diversidad de estudiantes que la institución tiene, por lo tanto se toma algunos aspectos relevantes del currículo para argumentar la viabilidad del proyecto de trabajo por áreas planteado en la Unidad Educativa Agronómico Salesiano. Entonces siendo la Autoridad Nacional la responsable del diseño curricular obligatorio, la 
Universidad Nacional Experimental Francisco de Miranda (UNEFM). Santa Ana de Coro. Venezuela

Mirian Carmita Arcentales-Fajardo; Darwin Gabriel García-Herrera; Nancy Marcela Cárdenas-Cordero; Juan Carlos Erazo-Álvarez

organización de la ejecución en torno al contexto le corresponde a la institución educativa, mediante su Proyecto Educativo Institucional (PEI) y el Plan curricular Institucional (PCI).

\section{Principios del currículo.}

El presente currículo está diseñado mediante destrezas con criterio de desempeño que tienen como meta que los estudiantes se apropien de manera integral de los conocimientos, habilidades y actitudes, que vienen en ellas, y puedan aplicar las mismas en situaciones concretas adaptadas y así mismo puedan hacerlo en situaciones diversas. Desde este punto de vista el proceso de enseñanza - aprendizaje debe darse desde todas las áreas de conocimiento, tomando en cuenta el aspecto interdisciplinar y multidisciplinar del conocimiento, que son importantes para enlazar y alcanzar una mejor comprensión de lo estudiado.

\section{Orientaciones metodológicas.}

El currículo propone que la metodología sea centrada en los estudiantes como actores principales, propiciando siempre el pensamiento racional y crítico, el trabajo individual y cooperativo dentro del aula, que motive la lectura y la investigación, los estudiantes deben alcanzar capacidades que permita cumplir los objetivos de aprendizaje planteados en el currículo, así mismo, se plantea que el trabajo docente debe ser colaborativo con el fin de fomentar el trabajo interdisciplinario y la coordinación de todo el equipo docente para que la atención al estudiante se desarrolle de la mejora manera.

\section{Autonomía de los centros para la concreción del Currículo.}

Considerando la autonomía y apertura que tienen las instituciones en cuanto a la parte pedagógica y la organización para el desarrollo del currículo, de acuerdo a su contexto social, cultural y las necesidades de los estudiantes, se creó la posibilidad de haber implementado el trabajo por áreas de conocimiento, con un docente para cada una de 
Universidad Nacional Experimental Francisco de Miranda (UNEFM). Santa Ana de Coro. Venezuela

Mirian Carmita Arcentales-Fajardo; Darwin Gabriel García-Herrera; Nancy Marcela Cárdenas-Cordero; Juan Carlos Erazo-Álvarez

ellas en la Unidad Educativa Agronómico Salesiano, con el objetivo de potenciar el proceso de enseñanza y aprendizaje de los estudiantes, el trabajo colaborativo docente y la calidad educativa institucional, esto analizado parcialmente en esta investigación.

\section{METODOLOGÍA}

La presente investigación es de tipo descriptivo no experimental, con un enfoque mixto y de cohorte transversal, que se efectuó en la Unidad Educativa Agronómico Salesiano, a los actores educativos, integrados por los Docentes de Básica Media, Estudiantes de Quinto, Sexto y Séptimo de Básica y a los Padres de Familia y/o Representantes legales de los niños y niñas del mismo nivel, para el estudio se tomó la totalidad de la población o universo que estaría conformada por 4 Docentes que imparten sus clases en cada área de conocimiento, 75 Estudiantes y 75 Padres de Familia representantes legales de este subnivel que muy gentilmente aceptaron participar en este trabajo.

La recolección de datos que sirvió para la elaboración de este documento se realizó de manera virtual en línea, mediante la plataforma zoom, dadas las circunstancias sanitarias del país, el proceso se desarrolló en tres momentos, primero se realizó una entrevista semiestructurada en grupo focal a los cuatro Docentes que imparten las clases en el subnivel de Básica Media, en un segundo momento se realizó el envío del link de la encuesta elaborada en Google Forms en escala de Likert, para los 75 estudiantes que integran este subnivel y de la misma manera en un tercer momento se envió el link de la encuesta para los Padres de Familia y representantes de los niños y niñas. El análisis estadístico se desarrolló con el programa IBM SPSS Statistics versión 19, proporcionado por la Universidad Católica de Cuenca de la cual se obtuvo las tablas y graficas presentadas en los resultados.

Se aplicó la encuesta como técnica de recoleccion de información, y dos instrumentos en escala de Likert, uno dirigido para los niños y niñas estudiantes de Básica Media, constituida por 10 preguntas y la segunda de 11 preguntas dirigida a los Padres de 
Universidad Nacional Experimental Francisco de Miranda (UNEFM). Santa Ana de Coro. Venezuela

Mirian Carmita Arcentales-Fajardo; Darwin Gabriel García-Herrera; Nancy Marcela Cárdenas-Cordero; Juan Carlos Erazo-Álvarez

familia, que fueron validadas a través del análisis de fiabilidad en la cual se obtuvo un alfa de Cronbach de 0.843 y de 0.914 respectivamente, las dos encuestas aunque con diferente enfoque recaban información sobre el nivel de satisfacción de la modalidad de trabajo en áreas de conocimiento, con docentes especialistas en el subnivel Básica Media.

Los instrumentos se aplicaron a un universo de 75 estudiantes de los cuales 70 respondieron representando un $93.3 \%$ del total y de la misma manera se aplica a 75 padres de familia de los cuales contestan 64 del subnivel antes mencionado representando un $85.33 \%$ del total; $y$, en el análisis de la normalidad de las variables que se realiza con un intervalo de confianza del 95\%, nos da como resultado que, absolutamente todas son paramétricas con un valor de $\mathrm{p}=0.000$ utilizando la prueba de Kolmogorov debido a que el universo supera los 50 datos analizados.

\section{RESULTADOS}

A continuación, se presentan análisis estadísticos relacionados con el uso de tablas de frecuencia y el Chi cuadrado que permiten evidenciar los resultados del trabajo planteado.

\section{Tabla 1.}

Se siente satisfecho al trabajar con un Docente para cada una de las áreas de Aprendizaje.

\begin{tabular}{lccccrr}
\hline & Frecuencia & Porcentaje & rrcentaje válido & $\begin{array}{r}\text { Porcentaje } \\
\text { acumulado }\end{array}$ \\
\hline Válidos & Muy insatisfecho & 1 & 1,4 & 1,4 & 1,4 \\
& Neutral & 4 & 5,7 & 5,7 & 7,1 \\
& Satisfecho & 22 & 31,4 & 31,4 & 38,6 \\
& Muy satisfecho & 43 & 61,4 & 61,4 & 100,0 \\
\cline { 2 - 6 } & Total & 70 & 100,0 & 100,0 & \\
\hline
\end{tabular}

Fuente: Elaboración Propia. 
CIENCIAMATRIA

Revista Interdisciplinaria de Humanidades, Educación, Ciencia y Tecnología

Año VI. Vol. VI. N³. Edición Especial III. 2020

Hecho el depósito de ley: pp201602FA4721

ISSN-L: 2542-3029; ISSN: 2610-802X

Universidad Nacional Experimental Francisco de Miranda (UNEFM). Santa Ana de Coro. Venezuela

Mirian Carmita Arcentales-Fajardo; Darwin Gabriel García-Herrera; Nancy Marcela Cárdenas-Cordero; Juan Carlos Erazo-Álvarez

En la tabla 1 se observó que el $61.4 \%$ de los estudiantes está muy satisfecho al trabajar con un Docente por asignatura en la Básica Media, motivo por el cual se debe mantener y fortalecer esta modalidad, mediante la motivación, capacitación y trabajo colaborativo de los docentes.

Tabla 2.

Relación entre ambiente de enseñanza y actividades docentes.

\begin{tabular}{|c|c|c|c|c|c|}
\hline & $\begin{array}{r}\text { Este ambie } \\
\text { escuela ayud }\end{array}$ & $\begin{array}{l}\text { de enseñar } \\
\text { mejorar su }\end{array}$ & $\begin{array}{l}\text { a en la } \\
\text { rendizaje }\end{array}$ & & \\
\hline & & Neutral & De acuerdo & $\begin{array}{l}\text { Totalmente } \\
\text { de acuerdo }\end{array}$ & Total \\
\hline Los docentes le proponen & Neutral & 0 & 1 & 1 & 2 \\
\hline $\begin{array}{l}\text { actividades diterentes que } \\
\text { le mantienen interesado }\end{array}$ & De acuerdo & 2 & 17 & 8 & 27 \\
\hline en las asignaturas & $\begin{array}{l}\text { Totalmente de } \\
\text { acuerdo }\end{array}$ & 3 & 16 & 22 & 41 \\
\hline Total & & 5 & 34 & 31 & 70 \\
\hline Pruebas de chi-cuadrado & & & & & \\
\hline & & Valor & $\mathrm{gl}$ & asintótica (b & \\
\hline Chi-cuadrado de Pearson & & $4,206^{a}$ & 4 & & 0,379 \\
\hline Razón de verosimilitudes & & 4,428 & 4 & & 0,351 \\
\hline Asociación lineal por lineal & & 1,521 & 1 & & 0,218 \\
\hline V de casos válidos & & 70 & & & \\
\hline
\end{tabular}

a. 5 casillas $(55,6 \%)$ tienen una frecuencia esperada inferior a 5. La frecuencia mínima esperada es 0,14.

Fuente: Elaboración propia. 
Universidad Nacional Experimental Francisco de Miranda (UNEFM). Santa Ana de Coro. Venezuela

Mirian Carmita Arcentales-Fajardo; Darwin Gabriel García-Herrera; Nancy Marcela Cárdenas-Cordero; Juan Carlos Erazo-Álvarez

Lo que demuestra la Tabla 2 analizada es, que, a pesar de tener una aceptación satisfactoria de las dos variables no hay una correlación directa, lo que significa que se debe plantear una propuesta de mejora en el aspecto de actividades de enseñanza planteada, y la interacción que estas promueven para mejorar el ambiente educativo.

Se realizó la prueba de Chi cuadrado, su resultado es de 0.379 , el cual es menor al nivel crítico establecido, por lo tanto, se acepta la hipótesis nula H0: No existe asociación entre la variable Este ambiente de enseñanza en la escuela ayuda a mejorar su aprendizaje; Los docentes le proponen actividades diferentes que le mantienen interesado en las asignaturas.

\section{Tabla 3}

La relación y el vínculo de afectividad y confianza con cada docente, hace que sus hijos sean más participativos e independientes en clase.

\begin{tabular}{llllll}
\hline & & & \multicolumn{2}{c}{ ircentaje válido } & Porcentaje \\
& & Frecuencia & Porcentaje & & acumulado \\
\hline Totalmente de acuerdo & 23 & 35,9 & 35,9 & 35,9 \\
\cline { 2 - 6 } & De acuerdo & 31 & 48,4 & 48,4 & 84,4 \\
\cline { 2 - 6 } Válidos & Neutral & 10 & 15,6 & 15,6 & 100,0 \\
\cline { 2 - 6 } & Total & 64 & 100,0 & 100,0 & \\
\cline { 2 - 6 } & & & &
\end{tabular}

Fuente: Elaboración propia.

En la tabla 3 los resultados demostraron que el $84.4 \%$ de los Padres de Familia están de acuerdo y totalmente de acuerdo con la relación afectiva que se establece entre los estudiantes y cada uno de los docentes de la Básica, porque esta hace que se mejore la actitud participativa y la independencia o autonomía de los niños y niñas de este nivel. 
CIENCIAMATRIA

Revista Interdisciplinaria de Humanidades, Educación, Ciencia y Tecnología

Año VI. Vol. VI. N³. Edición Especial III. 2020

Hecho el depósito de ley: pp201602FA4721

ISSN-L: 2542-3029; ISSN: 2610-802X

Universidad Nacional Experimental Francisco de Miranda (UNEFM). Santa Ana de Coro. Venezuela

Mirian Carmita Arcentales-Fajardo; Darwin Gabriel García-Herrera; Nancy Marcela Cárdenas-Cordero; Juan Carlos Erazo-Álvarez

Tabla 4.

Relación entre participación y seguimiento a estudiantes.

\begin{tabular}{|c|c|c|c|c|c|}
\hline \multicolumn{5}{|c|}{$\begin{array}{c}\text { Cree usted que sus hijos participan e interactúan más teniendo un docente por } \\
\text { asignatura. }\end{array}$} & \multirow[b]{2}{*}{ Total } \\
\hline & & Neutral & De acuerdo & $\begin{array}{c}\text { Totalmente de } \\
\text { Acuerdo }\end{array}$ & \\
\hline $\begin{array}{l}\text { Cree usted que la modalidad } \\
\text { implementada permite un }\end{array}$ & Neutral & 8 & 7 & 2 & 17 \\
\hline $\begin{array}{l}\text { mejor seguimiento a sus } \\
\text { representados y por lo tanto }\end{array}$ & De acuerdo & 6 & 17 & 10 & 33 \\
\hline $\begin{array}{l}\text { un mejor } \\
\text { aprendizaje. }\end{array}$ & $\begin{array}{l}\text { Totalmente de } \\
\text { acuerdo }\end{array}$ & 0 & 2 & 12 & 14 \\
\hline \multicolumn{2}{|c|}{ Total } & 14 & 26 & 24 & 64 \\
\hline \multicolumn{6}{|l|}{ Pruebas de chi-cuadrado } \\
\hline & & Valor & gl & \multicolumn{2}{|c|}{ Sig. asintótica (bilateral) } \\
\hline \multicolumn{2}{|c|}{ Chi-cuadrado de Pearson } & $23,689^{a}$ & 4 & \multicolumn{2}{|l|}{0,000} \\
\hline \multicolumn{2}{|c|}{ Razón de verosimilitudes } & 25,062 & 4 & \multicolumn{2}{|l|}{0,000} \\
\hline \multicolumn{2}{|c|}{ Asociación lineal por lineal } & 19,111 & 1 & \multicolumn{2}{|l|}{0,000} \\
\hline $\mathrm{N}$ de casos válidos & & 64 & & & \\
\hline
\end{tabular}

Fuente: Elaboración Propia.

En la Tabla 4. Se comprueba que el valor de $p=0.000$, siendo menor a 0.05 por lo tanto se rechaza la hipótesis nula, y se asume la correlación entre las variables: Cree usted que sus hijos participan e interactúan más teniendo un docente por asignatura; Cree usted que la modalidad implementada permite un mejor seguimiento a sus representados y por lo tanto un mejor aprendizaje, sin embargo

cabe resaltar que, a pesar de tener esta característica, se procedió a obtener un gráfico de apilados dentro del mismo programa IBM SPSS Stadistic y se obtiene que un 74, $5 \%$ están de acuerdo y totalmente de acuerdo con las variables planteadas, quedando la diferencia $25.5 \%$ que desconoce; para quienes se debe dar respuesta mediante el fortalecimiento de esta modalidad de trabajo con una propuesta de fortalecimiento que nos permita dar el alcance a todos los actores educativos. 
Universidad Nacional Experimental Francisco de Miranda (UNEFM). Santa Ana de Coro. Venezuela

Mirian Carmita Arcentales-Fajardo; Darwin Gabriel García-Herrera; Nancy Marcela Cárdenas-Cordero; Juan Carlos Erazo-Álvarez

\section{Tabla 5.}

Principales aportes de los Docentes entrevistados, en un análisis de la experiencia con la nueva modalidad de trabajo por Áreas en la Básica Media.

\begin{tabular}{lll}
\hline Unidad de Análisis & Respuestas \\
\hline Planificación y & Trabajo & $\begin{array}{l}\text { La planificación es más llevadera y promueve la creatividad del } \\
\text { Colaborativo en el aula. }\end{array}$ \\
& $\begin{array}{l}\text { docente para realizar clases más dinámicas, los docentes se } \\
\text { preparan más, se especializan y son más eficientes, se demuestra } \\
\text { solidaridad y trabajo compartido docente que se enriquece con la } \\
\text { experiencias de los otros. }\end{array}$ \\
\hline Organización y comunicación & $\begin{array}{l}\text { Se distribuye el tiempo de manera más efectiva y se puede dar } \\
\text { atención personalizada a los estudiantes, permite una mejor } \\
\text { interactividad, se puede decir que se establece una organización } \\
\text { de acuerdo a las necesidades de aprendizaje, } \\
\text { La comunicación es óptima permite alcanzar los objetivos. }\end{array}$ \\
\hline Aprendizaje & Habilidades & $\begin{array}{l}\text { Con esta modalidad se estimula el aprendizaje de manera positiva, } \\
\text { los estudiantes están a la expectativa de las actividades, y por ende } \\
\text { están más motivados, los niños reciben un mejor trato por parte de } \\
\text { los docentes, se adaptan para una nueva etapa, no les afecta la } \\
\text { transición al otro subnivel porque ya tienen experiencia y ello } \\
\text { potenciará su aprendizaje y habilidad social. }\end{array}$ \\
\hline
\end{tabular}

Fuente: Entrevista a Docente.

Luego de haber realizado la entrevista a los Docentes de Básica Media, evidenciamos que los resultados encontrados y sometidos al análisis como es el objetivo de esta investigación dan como resultado parcial que para la mayor parte de los actores educativos es una buena propuesta, a pesar de estar implementado desde el inicio del año lectivo 2020-2021, en la institución ha dado resultados que son significativos en el proceso educativo, no obstante se debe plantear una propuesta de fortalecimiento para esta modalidad de trabajo en la Básica Media. 
Mirian Carmita Arcentales-Fajardo; Darwin Gabriel García-Herrera; Nancy Marcela Cárdenas-Cordero; Juan Carlos Erazo-Álvarez

\section{PROPUESTA}

Una vez que se ha implementado el trabajo por áreas con Docentes para cada una de las asignaturas de la Básica Media, y luego de haber realizado el análisis con todos los actores educativos dentro de la investigación, se propone el "Fortalecimiento del ambiente educativo y las técnicas del trabajo colaborativo de los Docentes de la Básica Media"

\section{CEPID}

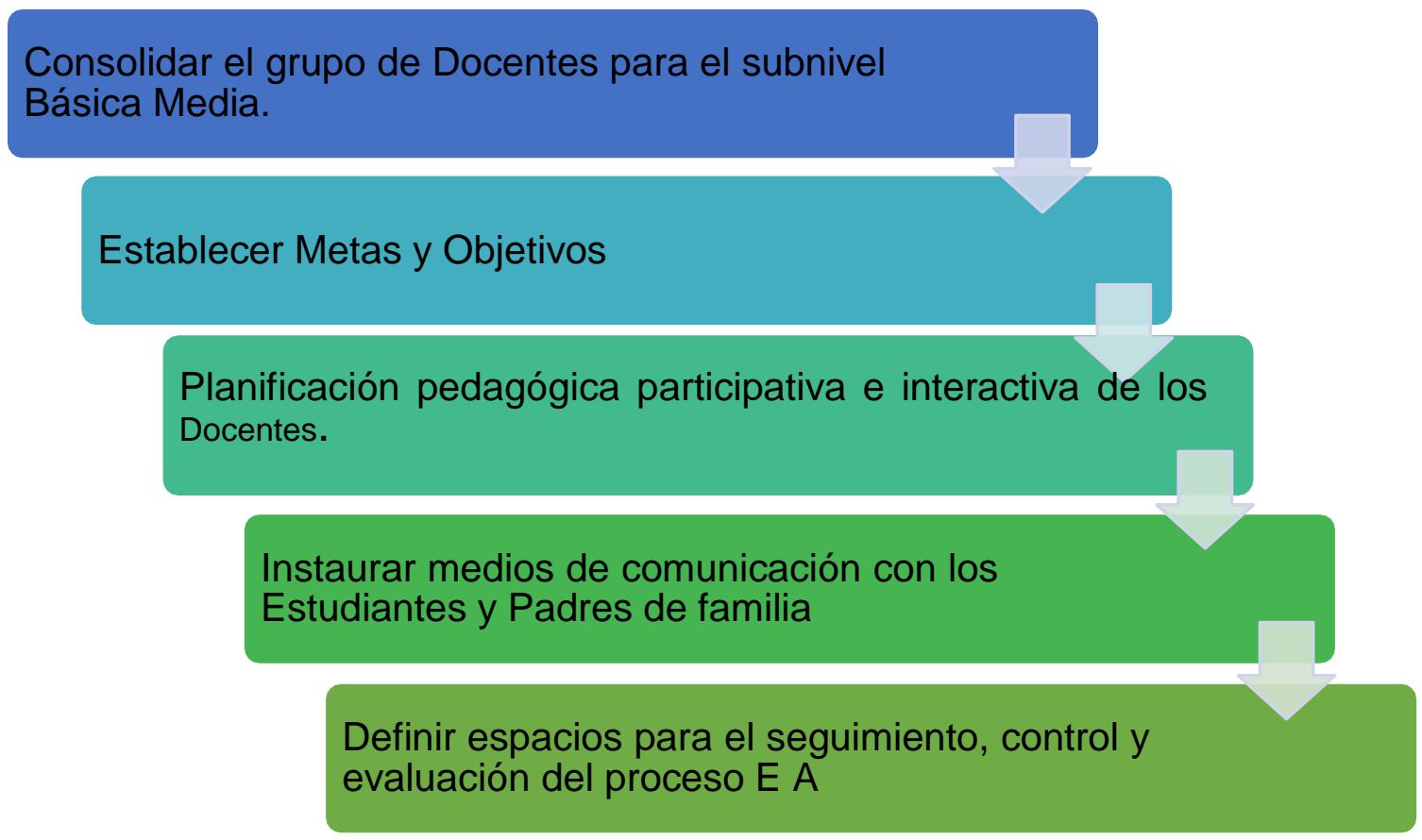

Figura 1. Esquema Fortalecimiento del ambiente educativo y las técnicas del trabajo colaborativo de los Docentes de la Básica Media.

Fuente: Elaboración propia. 
Universidad Nacional Experimental Francisco de Miranda (UNEFM). Santa Ana de Coro. Venezuela

Mirian Carmita Arcentales-Fajardo; Darwin Gabriel García-Herrera; Nancy Marcela Cárdenas-Cordero; Juan Carlos Erazo-Álvarez

Consolidar el grupo de Docentes para el subnivel de Básica Media: Para la propuesta de trabajo por áreas de conocimiento en este subnivel, la institución mediante su Líder Educativo, organiza el ambiente escolar para llevar a cabo el proyecto; es imprescindible que los docentes estén claros con la estructura implementada, se apropien de ella y se constituyan como un equipo que, con la utilización del Trabajo Colaborativo, desarrollen el proceso de enseñanza - aprendizaje.

Establecer Metas y Objetivos: Las metas y objetivos deben estar planteados en función de las necesidades de aprendizaje de los niños y niñas de este nivel, para alcanzar los aprendizajes que ellos requieren, con la utilización de metodologías activas, propendiendo a la autonomía y a alcanzar habilidades sociales que les servirá como antesala para los siguientes subniveles.

Planificación Pedagógica participativa e interactiva de los Docentes: La planificación debe estar enmarcada en la Propuesta Curricular Institucional, que considera los elementos que forman el currículo 2016, vigente para este y todos los niveles, su elaboración debe ser en conjunto de manera colaborativa, participativa, de tal forma que se pueda priorizar las DCD planear un trabajo interdisciplinario significativo desde y para todas las áreas de conocimiento.

Instaurar medios de comunicación con los Estudiantes y Padres de familia: La comunicación es importante dentro del proceso de aprendizaje, pues se promueve vínculos de confianza y apoyo desde todos los actores educativos, por lo tanto en la institución se utiliza la plataforma de gestión esemtia para la comunicación, esta herramienta permite una interactividad de información en tiempo real, con la cual los Estudiantes, Padres de Familia y Docentes están al tanto de todos los aspectos académicos, en estas circunstancias otro medio para establecer estos vínculos ha sido la plataforma Zoom, y de seguro en el momento que regresemos a las escuelas, también se realizara reuniones presenciales. 
Universidad Nacional Experimental Francisco de Miranda (UNEFM). Santa Ana de Coro. Venezuela

Mirian Carmita Arcentales-Fajardo; Darwin Gabriel García-Herrera; Nancy Marcela Cárdenas-Cordero; Juan Carlos Erazo-Álvarez

\section{Definir espacios para el seguimiento, control y evaluación del proceso de} Enseñanza Aprendizaje: En este periodo de tiempo luego de haber implementado esta nueva modalidad de trabajo en este subnivel, se ha realizado seguimientos de control para evaluar el impacto de la propuesta en los niños y niñas, resultando positivo en la mayor parte de los aspectos académicos, se plantea para esta evaluación hacer partícipes a los Padres de Familia, en reuniones con los Tutores de cada grado, para tener la perspectiva de ellos y considerar los aportes que sumen al proceso de aprendizaje y de esa forma llegar a la Calidad Educativa.

\section{CONCLUSIONES}

La investigación planteada e implementada en la institución, tiene como resultados parciales, que los estudiantes del subnivel básica media se adaptaron fácilmente a esta nueva dinámica de trabajo, sintiéndose motivados por la diversidad de actividades presentadas por los docentes, sus estrategias de enseñanza y la interactividad con ellos. A pesar de que la propuesta de trabajo por áreas y con Docentes para cada asignatura muestra una aceptación considerable por los actores educativos, según el análisis de chi cuadrado en la tabla 2, es susceptible de mejora, es así que se establece una propuesta en este sentido con el fin de fortalecer el proceso de enseñanza aprendizaje y potenciar las habilidades cognoscitivas y sociales de los niños y niñas de este subnivel.

La investigación en esta propuesta muestra un resultado satisfactorio desde la perspectiva de los Padres de Familia, que evidencia que sus hijos y/o representados sienten la cercanía y atención de los diferentes Docentes, obteniendo en consecuencia estudiantes con más confianza dentro de las aulas y por ende más participativos entre pares y en la relación Docente - Estudiante, creando un clima educativo de calidad.

Esta nueva propuesta implementada, ha mejorado la relación entre los Docentes de este subnivel promocionando el trabajo colaborativo, con el que se ha conseguido que los docentes compartan experiencias de enseñanza, materiales y recursos educativos, y 
Universidad Nacional Experimental Francisco de Miranda (UNEFM). Santa Ana de Coro. Venezuela

Mirian Carmita Arcentales-Fajardo; Darwin Gabriel García-Herrera; Nancy Marcela Cárdenas-Cordero; Juan Carlos Erazo-Álvarez

que, a partir de ello también se produzca un interaprendizaje en ellos y la producción de un trabajo interdisciplinario significativo en el aula de clase.

\section{REFERENCIAS}

Barrera Erreyes, H. M., Barragán García, T. M., \& Ortega Zurita, G. E. (2017). La realidad educativa ecuatoriana desde una perspectiva docente. [The Ecuadorian educational reality from a teaching perspective]. Revista Iberoamericana de Educación, 75(2), 9-20. Obtenido de https://rieoei.org/RIE/issue/view/242

Herrera Pavo, M. Á., \& Cochancela Patiño, M. G. (2020). Aportes de las reformas curriculares a la educación obligatoria en el Ecuador. [Contributions of curricular reforms to compulsory education in Ecuador]. Revista Scientific, 5(15), 362-383. Obtenido de https://doi.org/10.29394/Scientific.issn.2542-2987.2020.5.15.19.362$\underline{383}$

OREALC/UNESCO Santiago. (2013). UNESCO. Obtenido de https://n9.cl/3stnk

Amorim, A. (2017). Gestor escolar inovador: educação da contemporaneidade. [Director of the innovative school: contemporary education]. Revista Lusófona de Educação(35), 67-82. Obtenido de https://n9.cl/lkf7h

Bailey Moreno, J., Flores Fahara, M., \& González Rivera, P. E. (2016). Aprendizaje Docente y Reestructuración de sus creencias en los Procesos de Desarrollo Profesional y Gestión Escolar. [Teacher Learning and Restructuring of their beliefs in the Professional Development and School Management Processes]. Actualidades Investigativas en Educación, 16(3), 1-25. doi:10.15517/AIE.V16I3.25956

Castro Pérez, M., \& Morales Ramírez, M. E. (2015). Los ambientes de aula que promueven el aprendizaje, desde la perspectiva de los niños y niñas escolares. [Classroom Environments That Promote Learning from the Perspective of School Children]. Revista Electrónica Educare, 19(3), 1-32. doi:http://dx.doi.org/10.15359/ree.19-3.11

Cruz Aguilar, E. (2020). La educación transformadora en el pensamiento de Paulo Freire. [The transformative education]. Educere, 24(78), 197-206. Obtenido de https://n9.cl/28qlb 
Universidad Nacional Experimental Francisco de Miranda (UNEFM). Santa Ana de Coro. Venezuela

Mirian Carmita Arcentales-Fajardo; Darwin Gabriel García-Herrera; Nancy Marcela Cárdenas-Cordero; Juan Carlos Erazo-Álvarez

Escobar Medina, M. B. (2015). Influencia de la interacción alumno-docente en el proceso enseñanza-aprendizaje[Influence on the teaching-learning process between teacher-student]. Paakat: Revista de Tecnología y Sociedad, (8), 3-9. Obtenido de https://n9.cl/42n2n

Fuentes Sordo, O. E. (2015). La organización escolar. Fundamentos e importancia para la dirección en la educación. [School organization. Basis and relevance to direction on education]. Varona, (61), 1-12. Obtenido de https://n9.cl/9lnw4

González Vargas, J. E. (2014). Una Mirada del Trabajo Colaborativo en la Escuela Primaria desde las Representaciones Sociales. [A Look at Collaborative Work in Primary School from Social Representations]. Ra Ximhai, 10(5), 115-134. Obtenido de https://n9.cl/b6q8

Krichesky, G., \& Murillo, F. (2018). La Colaboración Docente Como Factor De Aprendizaje y Promotor[Teaching Collaboration as a Learning Factor and Promoter]. Educación XX1, 21(1), 135-155. doi:10.5944/educXX1.15080

Liesa, E., Castelló, M., \& Becerril, L. (2018). Nueva escuela, ¿nuevos aprendizajes?[New school, new learnings?]. REXE. Revista de Estudios y Experiencias en Educación, 2(1), 2-18. doi: https://doi.org/10.21703/

MINEDUC. (2020). Ministerio de Educación . Obtenido de https://educacion.gob.ec/

Ministerio de Educación. (2016). Ley orgánica de Educación Intercultural. Obtenido de https://n9.cl/1fo3

Ministerio de Educación. (2016). LOEl. Obtenido de https://n9.cl/1fo3

Ministerio de Educación. (2020). Educación General Básica Media. Obtenido de https://educacion.gob.ec/curriculo-media/

Oliveira Sá, S. (2017). A Interação entre pares: que lugar na avaliação do desempenho docente?"[The interaction between peers: the one used in the performance evaluation of the]. Revista Lusófona de Educação(37), 27-43. Obtenido de https://n9.cl/kj2m1 
Universidad Nacional Experimental Francisco de Miranda (UNEFM). Santa Ana de Coro. Venezuela

Mirian Carmita Arcentales-Fajardo; Darwin Gabriel García-Herrera; Nancy Marcela Cárdenas-Cordero; Juan Carlos Erazo-Álvarez

Ramírez-Cardona , C. A., Calderón-Hernández, G., \& Castaño-Duque, G. (2015). Enfoques Administrativos Presentes en Establecimientos Educativos. Un estudio empírico en instituciones colombianas de educación básica y media. [Administrative Approaches Present in Educational Establishments. An empirical study in Colombian institutions]. Revista Mexicana de Investigación Educativa, 20(66), 911-940. Obtenido de https://n9.cl/rc05

Zubizarreta, A. C., García - Ruiz, M. R., \& López , P. M. (2018). Impacto da prática nas crenças dos professores na formação sobre a relação família-escola. [Impact of the practicum on the beliefs of the teachers in training about the family-school relationship]. Revista Brasileira de Educação, 23. doi: 10.1590/S1413$\underline{24782018230028}$

(C2020 por los autores. Este artículo es de acceso abierto y distribuido según los términos y condiciones de la licencia Creative Commons Atribución-NoComercial-Compartirlgual 4.0 Internacional (CC BY-NC-SA 4.0) (https://creativecommons.org/licenses/by-nc-sa/4.0/) 